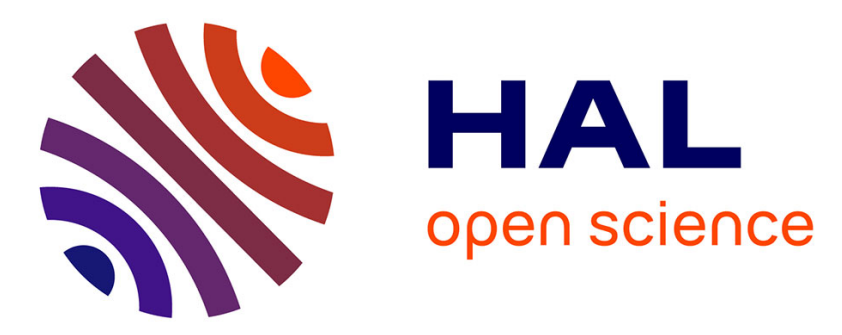

\title{
Kin competition drives the evolution of sex-biased dispersal under monandry and polyandry, not under monogamy
}

Thomas Brom, Manuel Massot, Stéphane Legendre, David Laloi

\section{- To cite this version:}

Thomas Brom, Manuel Massot, Stéphane Legendre, David Laloi. Kin competition drives the evolution of sex-biased dispersal under monandry and polyandry, not under monogamy. Animal Behaviour, 2016, 113, pp.157-166. 10.1016/j.anbehav.2016.01.003 . hal-01297762

\section{HAL Id: hal-01297762 https: / hal.sorbonne-universite.fr/hal-01297762}

Submitted on 4 Apr 2016

HAL is a multi-disciplinary open access archive for the deposit and dissemination of scientific research documents, whether they are published or not. The documents may come from teaching and research institutions in France or abroad, or from public or private research centers.
L'archive ouverte pluridisciplinaire HAL, est destinée au dépôt et à la diffusion de documents scientifiques de niveau recherche, publiés ou non, émanant des établissements d'enseignement et de recherche français ou étrangers, des laboratoires publics ou privés. 
5 Manuel Massot*

6 Stéphane Legendre $†$

7 David Laloi*

8 *: Sorbonne Universités, UPMC Univ Paris 6, CNRS, UMR 7618, Institut d'écologie et des

9 sciences de l'environnement de Paris (iEES Paris), Paris, France

\section{monandry and polyandry, not under monogamy}

†:UMR 8197 IBENS (CNRS, ENS), École Normale Supérieure

Received 1 October 2015

Initial acceptance 11 November 2015

Final acceptance 21 December 2015

MS. number: 15-00843R

Correspondence: T. Brom, Université Pierre et Marie Curie, Institut d'écologie et des sciences

de l'environnement de Paris, case 237, 7 quai Saint Bernard, 75005 Paris, France.

E-mail address: thomas.brom@upmc.fr

The relation between mating system and sex-biased dispersal has been debated for three decades. However, the relative importance of the processes involved in this relation remains

1 poorly known. In this study, we paid special attention to kin competition. We built an

2 adaptive individual-based model fixing three mating systems (monandry, polyandry,

3 monogamy) in a metapopulation, and allowing dispersal across patches to evolve

4 independently for males and females. Our simulations showed that a difference in the number

5 of mates can determine the evolution of sex-biased dispersal. Dispersal appears strongly male 
26 biased under monandry and polyandry, but balanced under monogamy. By contrast, we

27 showed that inbreeding can influence but does not promote sex-biased dispersal, and that the

28 primary sex ratio does not qualitatively affect the evolution of sex-biased dispersal under

29 monandry and polyandry. These results are driven by the interaction of two factors: the

30 variation in reproductive success between patches in the metapopulation and kin competition.

31 These two factors are influenced by the mating system, which modifies both the competition

32 for access to partners and the mean relatedness between individuals. To ascertain that kin

33 competition actually drives sex-biased dispersal, we made simulations with destruction of any

34 genetic structure in the metapopulation, and we found that in this case dispersal was not sex

35 biased.

36

37

38 Keywords: dispersal, individual-based model, kin competition, mating system, sex-biased 39 dispersal, spatial heterogeneity.

40 
Mating and dispersal are two key events in the life of an individual, which are thought

to be linked by several mechanisms (Greenwood, 1980; Gros, Poethke, \& Hovestadt, 2009;

Perrin \& Goudet, 2001; Perrin \& Mazalov, 2000). In particular, the link between mating system and dispersal has been largely invoked to explain sex-biased dispersal (Chapple \& Keogh, 2005; Greenwood, 1980; Mabry, Shelley, Davis, Blumstein, \& van Vuren, 2013;

Nagy, Günther, Knörnschild, \& Mayer, 2013), the most discussed feature in the dispersal

literature. The main hypothesis suggests that prevailing male dispersal, as observed in mammals, and prevailing female dispersal, as observed in birds, are due to the predominance of polygyny and monogamy in each group, respectively (Greenwood, 1980). However, more than 30 years after Greenwood proposed this hypothesis, it is still debated (Dobson, 2013; Johnson \& Gaines, 1990; Mabry et al., 2013). Some evidence exists that links the mating system and sex-biased dispersal, especially in mammals (Mabry et al., 2013), but it remains challenging to conclude whether the mating system actually drives the evolution of dispersal or whether these two traits only covary (Clutton-Brock \& Lukas, 2012). Some modelling results suggest that the mating system alone can indeed drive the evolution of dispersal (Perrin \& Goudet, 2001; Perrin \& Mazalov, 1999, 2000). However, because dispersal is influenced by many parameters (Clobert, Massot, \& Le Galliard, 2012), it is often difficult to determine whether its evolution depends on the mating system itself or on other life history traits correlated with the mating system (Lawson Handley \& Perrin, 2007).

Dispersal is not only widespread in life (Dobson, 1982), it is also a highly multifaceted trait. Primarily, there can be natal or breeding dispersal (Dobson, 2013), as well as short- or long-distance dispersal (Murrell, Travis, \& Dytham, 2002). In motile animals, dispersal appears as a complex process with several phases, such as departure, transfer and settlement, rather than a holistic behaviour (Clobert, Le Galliard, Cote, Meylan, \& Massot, 2009; Matthysen, 2012). To investigate its fundamental effects on the genetic structure of 
populations and metapopulations (Kokko \& López-Sepulcre, 2006; Travis \& Dytham, 1998), dispersal is often defined as 'any movement of individuals or propagules with potential consequences for gene flow across space' (Ronce, 2007, p.232). Beyond genes, dispersal can also impact social interactions (Boudjemadi, Lecomte, \& Clobert, 1999) and demography (Massot, Clobert, Pilorge, Lecomte, \& Barbault, 1992). For these reasons, dispersal is a key determinant of population persistence in the context of habitat fragmentation (Parvinen, Dieckmann, Gyllenberg, \& Metz, 2003), climate warming (Clobert et al., 2009; Walther et al., 2002) and the success of invasive species (Kubisch, Fronhofer, Poethke, \& Hovestadt, 2013). It is generally assumed that dispersal is costly, which includes the predispersal cost of development of dispersal-related traits, as well as the cost in time, energy and exposure to various risks during dispersal (Bonte et al., 2012). These costs can negatively affect survival or reproductive success of dispersers. Thus, individuals should disperse only if sufficient benefits of dispersal outweigh its costs. The fitness benefits of dispersal often result from the avoidance of local costs related to habitat quality, inbreeding or competition (Clobert, Danchin, Dhondt, \& Nichols, 2001; Clobert et al., 2012). Particular attention has been paid to the relative importance of the costs and benefits of dispersal for males and females, which can be affected by the corresponding costs and benefits related to the mating system. For instance, dispersal can depend on the relationship between the mating system and competition for mates or for resources to attract mates. Investment in mating, and in obtaining associated resources, varies between males and females depending on the mating system. In monogamy, males compete for resources needed to feed offspring, whereas in polygyny, males compete more directly for mating opportunities but less for resources. Therefore, competition for resources is more challenging for males in monogamous species and for females in polygynous species. Because dispersal is thought to reduce the competitive ability to access 
resources (Massot, Clobert, Lecomte, \& Barbault, 1994), higher dispersal should be selected for females in monogamous systems and males in polygynous systems (Greenwood, 1980). The genetic environment has been shown to play a key role in the evolution of dispersal. In particular, inbreeding avoidance has often been put forward to explain sex-biased dispersal. In contrast, kin competition avoidance has been somewhat neglected (Dobson, 2013), despite some theoretical (Hamilton \& May, 1977; Poethke, Pfenning, \& Hovestadt, 2007) and empirical evidence (Clobert et al., 2012; Lambin, Aars, \& Piertney, 2001). First, Hamilton and May (1977) showed, using a game-theoretical approach, that dispersal can evolve in response to kin competition, even if the intensity of competition is constant in space. Then, Frank (1986) showed that dispersal equilibrium can be directly linked to relatedness, and thus kin competition. Taylor (1988) extended this work, showing in particular that haplodiploidy promotes sex-biased dispersal. In a highly cited paper, Perrin \& Mazalov (2000) investigated sex-biased dispersal following Greenwood's (1980) mating system hypothesis and showed that sex-biased dispersal evolution is possible when males and females do not compete in the same way for resources. However, this kind of deterministic model can miss stochastic effects as pointed out by Gros et al. (2009). Using an individualbased model (stochastic by construction), these authors put forward another mechanism to explain sex-biased dispersal. They showed that sex-specific spatiotemporal variance of fitness between patches in the metapopulation can promote sex-biased dispersal. However, they did not unravel the role of kin competition, and they contrasted a random mating with a harem system, which would magnify the expected effect.

In this study, we investigated the interplay of kin competition and spatiotemporal variance of fitness on the evolution of sex-biased dispersal. We considered the influence of genetic mating system on the coevolution of male and female dispersal behaviour in a metapopulation. We modelled three genetic mating systems, monogamy, monandry and 
115 polyandry, which differed only in the number of partners that females and males can have.

116 We focused on how these three mating systems, having different impacts on the genetic 117 structure of the metapopulation, and different levels of competition for mates, can affect

118 dispersal in each sex. We assessed quantitatively the effect of mating system on the dispersal 119 rate in relation to important parameters such as the sex ratio and the intensity of inbreeding 120 depression. We used an individual-based model, where dispersal was adaptive, to take into 121 account kin selection and stochasticity. We estimated the relative importance of kin selection 122 versus individual selection by breaking the genetic structure of the metapopulation using the 123 method of Poethke et al. (2007).

124

$<$ H1 $>$ The model

In our adaptive individual-based model (Bach, Thomsen, Pertoldi, \& Loeschcke, 2006) of dispersal evolution, mainly inspired by the work of Poethke et al. (2007), dispersal is the only adaptive trait. We did not compute individual or inclusive fitness; these, as well as kin interactions, were emergent properties of the simulations. The evolutionarily stable 130 dispersal rate was also an outcome of the simulations. $<H 2>$ Demography

132 The model considers populations of sexually reproducing diploidic organisms occupying a 133 number $n_{\text {patch }}$ of patches and constituting a metapopulation. The model follows a simple life 134 cycle with, in order, dispersal, mating, reproduction, birth, survival. Adults die after 135 reproduction so that generations do not overlap. All patches have the same carrying capacity $K$, but the reproductive quality of patches 137 varies along time and across space. Patch quality is drawn from a log-normal distribution with 138 mean $\lambda$ and standard deviation $\sigma$. Therefore $\sigma$ describes the heterogeneity in patch quality. 
139 The quality of a patch $j$ at time $t$ is $\Lambda(t, j)$. The fecundity $F_{i}$ of each female $i$ in patch $j$ at 140 time $t$ is drawn from a Poisson distribution with mean $\Lambda(t, j)$. The sex of newborns is 141 determined by inheritance of the sexual chromosomes of their parents (XY model). The sex 142 ratio at birth, $s r_{\mathrm{b}}$, is balanced in most simulations, but can be biased for exploratory purposes 143 by changing the probability of the inheritance of sexual chromosomes. The realized sex ratio 144 at birth is $n_{\text {males }} /\left(n_{\text {females }}+n_{\text {males }}\right)$, with $n_{\text {males }}$ and $n_{\text {females }}$ the counted number of 145 newborns in each sex. Its average value is equal to the probability $s r_{\mathrm{b}}$ of inheriting the 146 father's Y chromosome and becoming a male. Newborns survive and reach the dispersal phase with the density-dependent survival 148 probability

$$
s=\frac{1}{\left(1+\alpha N_{j}\right)^{\beta}}
$$

149 where $\alpha=\left(\lambda^{1 / \beta}-1\right) / K, N_{j}$ is population size in patch $j$ and $\beta$ parameterizes the intensity 150 of density dependence (Poethke et al., 2007). The survival probability decreases with 151 increasing $N_{j}$, and decreases faster for small $\beta$. Note that $N_{j}$ includes only newborns because 152 adults die after reproduction. $<H 2>$ Mating

154 The success of a female depends on patch quality, the survival of its offspring and its mating 155 with at least one male. The mating success of a male depends on the female(s) it mates with. 156 We investigated three mating systems; in each, pairs were formed from males and females 157 drawn randomly within their patch. (1) In monandry each female mates with only one male 158 and males have no reproduction limit. (2) In polyandry each female mates with many males, 159 males have no reproduction limit and each newborn has a father chosen randomly in the 160 patch. (3) In monogamy each female mates with only one male and males are no longer 
161

162

163

164 165 166

available after a single mating. Thus, males and females have a single partner, and some individuals do not reproduce when the breeding sex ratio is unbalanced in a patch.

\section{$<H 2>$ Dispersal}

To allow for sex-specific dispersal, males and females are endowed with two independent loci $\left(d_{\mathrm{m}}\right.$ and $\left.d_{\mathrm{f}}\right)$ that drive dispersal independently in each sex. Each newborn inherits two dispersal alleles, one randomly chosen from its mother and one randomly chosen from its father. Mutations occur with frequency $f_{\mathrm{s}}$, the new value of a mutated allele being drawn from a normal distribution with mean equal to the value of the ancestor allele and standard deviation $s d_{\mathrm{s}}$. The dispersal strategy $d s_{i}$ of an individual $i$ is identified with the mean values of the two alleles expressed by its sex. The dispersal probability of the individual $d p_{i}$ depends on density as follows:

$$
d p_{i}=\left\{\begin{array}{c}
0 \text { if } N_{j} / K \leq d s_{i} \\
1-\frac{d s_{i}}{\mathrm{~N}_{j} / K} \text { if } N_{j} / K>d s_{i}
\end{array}\right\} .
$$

173 Following this equation, we can see that the dispersal strategy $d s_{i}$ acts as a threshold: if the 174 patch density $N_{j} / K$ is under the threshold, the individual never disperses; if the patch density 175 is above the threshold, the higher the density, the higher the probability of dispersing. 176 Therefore, whenever the patch density is near the dispersal threshold, the dispersal probability 177 will be very low. Dispersal is global, toward a randomly selected patch. The cost of dispersal 178 is modelled by a probability $\mu$ of dying during dispersal. In most simulations this cost is 179 identical for males and females, but we also tested the effect of a sex-biased cost. $<H 2>$ Relatedness, inbreeding and heterozygosity

181 Each individual is given 32 diploid neutral (not under natural selection) loci, with each locus 182 having two different alleles, A and B. For each allele of a neutral locus, the process of 183 inheritance is the same as for dispersal alleles, and mutations occur with frequency $f_{n}$. 184 Mutation performs a switch between the two alleles. This set of loci allows us to measure the 
relatedness between two individuals at the population and metapopulation levels, taking into account relatedness and possible changes in population size. The heterozygosity of an individual is calculated by assessing the heterozygosity at each locus and counting the heterozygous loci relative to the total number of loci. A fully homozygous individual $i$ has a heterozygosity level $H_{i}$ of 0 . A fully heterozygous individual has a heterozygosity level of 1 . On average, the heterozygosity level of an individual decreases with increasing relatedness between its parents. Therefore, we use the heterozygosity level to model inbreeding depression. Homozygous females can suffer a fecundity loss reducing the initial fecundity:

$$
F_{i}=\left\{\begin{array}{c}
F_{i} \text { if } H_{i} \geq 0.5 \\
F_{i} \times\left(2 H_{i}\right)^{\rho} \text { if } H_{i}<0.5
\end{array}\right\}
$$

where $H_{i}$ is the heterozygosity level of female $i$ and $\rho$ is the strength of the inbreeding penalty. When $\rho$ is equal to 0 , there is no homozygosity depression. When $\rho>0$, females suffer a loss in fecundity that increases with $\rho$.

\section{$<H 2>$ Test on kin competition}

As said before, kin interactions are emergent properties in an individual-based model. However, as in Poethke et al. (2007), we performed simulations in a shuffled version of the model to cancel kin competition. In this shuffled model, before dispersal occurs, individuals are randomly redistributed in the whole metapopulation, but preserving the initial patchspecific densities and sex ratios. Therefore, the genetic structure is broken, but the demographic structure remains unchanged. In the unshuffled simulations, a dispersing individual has less chance of competing with kin in its patch of arrival than in its patch of departure. In the shuffled simulation, because individuals are randomly redistributed before dispersal, the chance of competing with kin is the same across all patches. Thus, the comparison between the shuffled and the unshuffled simulations allows to test specifically the effect of kin competition on the evolution of male and female dispersal. 
The simulation parameters used are reported in Table 1. Our results were based on the final

210 outcome of many runs for each parameter set (Monte Carlo simulation). We made sure that

211 equilibrium was reached by letting the simulations run a large number of generations (15 000

212 at least) and verified that the mean dispersal rate was stable for each sex. We did not use

213 statistical significance tests that are inappropriate to compare simulation model results (White,

214 Rassweiler, Samhouri, Stier, \& White, 2014). We followed the two arguments of White et al.

215 (2014): first, the potentially infinite number of replications can artificially increase the power

216 of statistical tests. Second, two sets of simulations with different parameters lead to different

217 outcomes. Thus, we focused our analysis on the magnitude of the difference between

218 simulations. Our results are shown with 95\% confidence intervals to ensure that a difference

219 between two sets of simulations is not the result of stochasticity.

\section{$<$ H1 $>$ Results}

\section{$<H 2>$ Mating system and local relatedness}

Our simulations show that the mating system influences the evolution of sex-biased dispersal. Males and females evolve the same dispersal rate in monogamy, while the dispersal rate is higher in males in the monandrous and polyandrous mating systems (Fig. 1). Moreover, males disperse more in monandry than in polyandry. In the shuffled simulations, where the effect of kin competition is removed, a lower dispersal rate evolves in both sexes and the male bias in dispersal disappears (Figs 1 and 2).

The difference in dispersal between males and females in monandry and polyandry is the result of the interplay of two processes. First, there is a strong kin competition effect, as revealed by the lower dispersal in both sexes in the shuffled simulations (Figs 1 and 2). This effect is expected to be stronger in monandry than in polyandry because a smaller proportion of males reproduce in monandry, so that local relatedness is higher (illustrated in Fig. A1). Second, the mating system creates an asymmetry between the sexes in the spatiotemporal 

between patches is the same for males and females in monogamy, whereas this variation is higher for males in monandry and polyandry (Fig. A2). This sex bias in the variability of

variability of reproductive success between patches. The variability of reproductive success reproductive success persists in the shuffled simulations, i.e. when there is no kin competition (Fig. A2).

Inbreeding also affects dispersal. Increasing the penalty of homozygosity (i.e. the cost of inbreeding) increases the dispersal rate, but this average effect also depends on the mating system (Fig. 3). Under monogamy, both sexes evolve higher dispersal rates with increasing homozygosity penalty. Under polyandry and monandry, the increase in dispersal with higher homozygosity penalty occurs mainly in males.

\section{$<H 2>$ Heterogeneity in patch quality, dispersal cost and sex ratio}

Dispersal increases with increasing heterogeneity in patch quality, and sex-biased dispersal is reduced for high heterogeneity in patch quality (Fig. 2). At the same time, dispersal rate decreases with increasing dispersal cost in both sexes, and sex-biased dispersal only appears when the mortality of dispersers is lower than $25 \%$ (Fig. A3). We also tested the effect of sex-biased dispersal cost (Fig. 4). We changed the male dispersal cost keeping the female dispersal cost unchanged. As expected, a sex-biased dispersal cost modifies the evolution of sex-biased dispersal. Whatever the mating system, an increased male dispersal cost decreases male dispersal rate. Consequently female dispersal increases as male dispersal decreases. In monogamy, the sex with the higher dispersal cost has the lower dispersal rate at equilibrium. In monandry and polyandry, dispersal is female biased for high values of male dispersal cost (e.g. in Fig. 4, female-biased dispersal evolves when male dispersal cost is about $30 \%$ higher than female dispersal cost). 
A bias in the primary sex ratio has different effects depending on the mating system

259 (Fig. 5). In monogamy, a bias in sex ratio induces sex-biased dispersal: the more numerous sex disperses more. Although male dispersal still increases under monandry and polyandry when the sex ratio is male biased, a bias in sex ratio does not qualitatively change the sex bias in dispersal. In other words, the primary sex ratio does not appear to affect the evolution of sex-biased dispersal in the monandrous and polyandrous mating systems.

\section{$<$ H1 $>$ Discussion}

The importance of kin competition in the evolution of dispersal has been well established by several theoretical works (Comins, Hamilton, \& May, 1980; Hamilton \& May, 1977; Poethke et al., 2007; Taylor, 1988). The situation is more contrasted with regard to the evolution of sex-biased dispersal. For instance, Perrin and Mazalov (2000) have shown that male-biased dispersal can evolve in polygynous/promiscuous mating systems in response to

271 kin competition. However, they did not take into account stochastic effects and used an 272 unrealistic exponential growth assumption (Gros et al., 2009). More recently, Lehmann \& 273 Balloux (2007) developed an analytical model taking into account both kin competition and 274 spatiotemporal variance in fecundity, but they did not address the question of mating process 275 nor the coevolution of male and female dispersal behaviour. In the present study, we have built an individual-based model to investigate the effect

277 of different mating systems, defined in our study by the number of mates, on the evolution of 278 sex-biased dispersal through their influence on kin competition. We revealed the role of kin 279 competition by contrasting models with or without genetic structure, i.e. with or without 280 indirect fitness benefits of kin competition avoidance by dispersal. Our model is focused only 281 on intragenerational kin competition and does not include parent-offspring conflict or kin 282 cooperation behaviours (Perrin \& Lehmann, 2001), i.e. the other two kin-related processes 
283 often cited as being involved in dispersal evolution (Lambin et al., 2001). We showed that 284 intragenerational kin competition can play a central role in the evolution of sex-biased 285 dispersal, and that it can be, under a large range of conditions, a better candidate than 286 inbreeding risk. Therefore, our results confirm the role of kin competition in dispersal 287 evolution and bring new insights to its role in the evolution of sex-biased dispersal. Mainly, 288 we showed that, when the primary sex ratio and dispersal costs are balanced, sex-biased 289 dispersal does not evolve in the absence of genetic structure, i.e. in the absence of kin-related 290 benefit to disperse. We thus pointed out the importance of kin competition avoidance in the evolution of sex-biased dispersal (Figs 1 and 2). We observed a higher male bias in dispersal under monandry than polyandry (Fig. 1). Furthermore, we found that heterogeneity in patch 293 quality, dispersal cost, inbreeding and primary sex ratio also affected the evolution of sex294 biased dispersal. These factors can modulate the influence of the mating system. In our model, the influence of the mating system can be explained by the interaction between two phenomena. First, kin competition affects both sexes, but its effect is stronger in monandry than in polyandry due to a higher local relatedness (Fig. A1), a consequence of a 298 smaller proportion of males that reproduce in monandry. Second, in monandry and polyandry, 299 males obtain higher benefits of dispersal because they experience a higher variance in their 300 reproductive success between patches than females (Fig. A2) as described in Gros et al. 301 (2009). In monogamy, males and females are subjected to the same competition processes; 302 thus they experience the same variance in reproductive success and disperse equally. In 303 polyandry and monandry, fewer males reproduce than females, and this difference between 304 the sexes is even more pronounced in monandry. Therefore, the variance in reproduction 305 between patches is higher for males than females, and higher in monandry than polyandry. 306 This difference between males and females, in interaction with strong enough kin 307 competition, leads to the evolution of male-biased dispersal (Figs 1, A1, A2). As in Perrin and 
Mazalov (2000), our results show that mating system and kin competition influence sex-

309 biased dispersal. However, the mechanisms at stake in our simulations differ from those 310 proposed by Perrin and Mazalov. Our results show the evolution of male-biased dispersal 311 without relaxing kin competition in females, and the influence of the variance in reproduction 312 between patches. In addition, we did not limit our modelling to the assumption of exponential 313 growth.

Both kin competition and the variance in reproductive success can be affected by other factors and by the feedback of dispersal. For example, high heterogeneity in patch quality has two effects: first, as widely found, it induces the evolution of a high dispersal rate (Bach et al., 2006; Gros, Hovestadt, \& Poethke, 2008; Poethke et al., 2007; Travis \& Dytham, 1998) that reduces kin competition; second, it reduces sex bias in the variance of reproductive success.

319 These two effects lower the difference between male and female benefits of dispersal and ultimately lower the sex bias in dispersal. Dispersal cost also has an influence on sex-biased dispersal. A very low or very high dispersal cost reduces the sex bias in dispersal (Fig. A3). A potentially important element of the interaction between individual benefit, kin benefit and 323 dispersal cost is the dispersal decision rule. In our model, we used density-dependent 324 dispersal, and, therefore, individuals have information on their potential dispersal benefits 325 (Clobert et al., 2009). However, density-independent dispersal simulations led to the same evolution of sex-biased dispersal in the three mating systems studied, with the same evidence 327 of the key role of kin competition (Fig. A4). The effect of kin competition on the evolution of sex-biased dispersal can be affected 329 by the biological and ecological characteristics of organisms. Most of the hypotheses on sex330 biased dispersal were proposed to explain dispersal patterns in birds and mammals, which

331 show mainly female-biased and male-biased dispersal, respectively (Dobson, 2013; 332 Greenwood, 1980). The main hypothesis, which relates sex-biased dispersal and the 
333 preponderant mating system in each of these two groups, remains under debate (Mabry et al.,

334 2013). Our model can adapt to different organisms, but our parameterization fitted better with 335 the biology of invertebrates. Invertebrates include organisms with very variable biological and 336 ecological traits, but most of them suffer a high dispersal cost and also have a high fecundity 337 (Benton \& Bowler, 2012). We can expect a high fecundity (with a large variance) to induce a 338 high heterogeneity across patches that should often cancel sex bias in dispersal (Fig. 2). An 339 interesting case, according to our results, is provided by Markow and Castrezana (2000) who 340 found no sex-biased dispersal in two Drosophila species and a male-biased dispersal in a 341 third. The latter species showed a stronger population genetic structure and a lower dispersal 342 rate than the other two. This result is in accordance with our predictions. Sex-biased dispersal 343 was also found in other species, such as a male-biased dispersal in a butterfly (Bennett, Pack, 344 Smith, \& Betts, 2013), a ground beetle (Lagisz, Wolff, Sanderson, \& Laskowski, 2010), a 345 neotropical orchid bee (López-Uribe, Zamudio, Cardoso, \& Danforth, 2014) and a female346 biased dispersal in damselflies (Beirinckx, Van Gossum, Lajeunesse, \& Forbes, 2006). As 347 mentioned by Benton and Bowler (2012), invertebrates often lay many eggs in a small area 348 and should then suffer strong kin competition, which could explain the evolution of sex349 biased dispersal. In addition, as illustrated by our results, a better understanding of sex-biased 350 dispersal and of the effect of the mating system requires us to pay attention to other 351 parameters such as kin interactions, inbreeding, dispersal cost, intensity of local competition 352 (for resources, mates), genetic structure and sex ratio. This broader approach is also justified 353 by the accumulating evidence of the multideterminism of dispersal (Clobert et al., 2012) and 354 seems useful to explain sex-biased dispersal (Lambin et al., 2001). As already mentioned, the mating system is central to explain sex-biased dispersal in many species. Usually, mating systems are characterized by the number of mates of each 357 individual and the defence of mating resources (Reynolds, 1996). In our simulations, we 
358 investigated the effect of the number of mates. In the three different mating systems

359 (monandry, polyandry, monogamy), our results never showed a female-biased dispersal when

360 sex ratio is balanced and cost of dispersal unbiased. We also independently tested the defence

361 of mating resources via unequal dispersal costs between males and females, an important

362 hypothesis to explain sex-biased dispersal (Greenwood, 1980; Gros et al., 2008). For example,

363 males that compete for territory may pay a high cost when they disperse because they lose

364 information on their local environment. In this case, females should have a higher dispersal 365 rate than males because they do not pay this cost (Fig. 4). Results obtained in the Siberian jay, 366 Perisoreus infaustus, by Gienapp and Merilä (2011) agree with this hypothesis. Other 367 differences between male and female dispersal costs were identified in birds (Nevoux, Arlt, 368 Nicoll, Jones, \& Norris, 2013), mammals (Soulsbury, Baker, Iossa, \& Harris, 2008) and 369 invertebrates (Gu, Hughes, \& Dorn, 2006; Nespolo, Roff, \& Fairbairn, 2008). Local relatedness affects not only kin competition but also inbreeding. Inbreeding is a

371 key factor historically proposed to explain the evolution of sex-biased dispersal (Dobson, 372 2013). Previous deterministic models have shown that inbreeding is a good candidate to 373 explain sex-biased dispersal in the absence of kin competition, but it has weaker effects when 374 kin competition is taken into account (Perrin \& Goudet, 2001). In the same way, our results 375 indicate that inbreeding is more able to reinforce an existing sex bias in dispersal than to 376 create such a bias. Whereas our model predicts a strong influence of kin competition, 377 inbreeding does not qualitatively change the results and does not promote sex-biased 378 dispersal. This is in agreement with Guillaume and Perrin (2006), although these authors 379 modelled the genetic load in a different way. Interactions between kin competition and 380 inbreeding are complex, and in many theoretical cases adding inbreeding does not affect 381 dispersal evolution (Roze \& Rousset, 2005). 

different groups can modify the primary sex ratio of their offspring (Alonso-Alvarez, 2006; interesting to draw a parallel between sex-biased dispersal and sex-biased sex ratio because both processes can evolve in response to the same factors (Leturque \& Rousset, 2004; West et al., 2005). We did not model the coevolution of sex ratio adjustment and dispersal (see Wild \& Taylor, 2004), but we tested the effect of a change in the sex ratio on sex-biased dispersal under different mating systems (Fig. 5). Our results showed, as expected, a strong influence of the sex ratio under monogamy because the number of available partners is crucial in this mating system. By contrast, there was only a quantitative effect of the sex ratio on sex-biased 392 dispersal under monandry and polyandry: even a strongly female-biased sex ratio did not lead 393 to the evolution of a high female dispersal nor reduce the bias towards male dispersal. If we 394 had built our model with a limited number of reproductive places by patch, as in some other 395 models (Gros et al., 2008, 2009; Perrin \& Mazalov, 2000; Wild \& Taylor, 2004), the sex ratio 396 would have had a higher effect because of the competition between females for these places. To conclude, using a model where we defined mating systems by the number of mates, 398 we found that the mating system influences the evolution of sex-biased dispersal through both 399 the pair bond pattern and the genetic structure of the population, giving a key role to kin 400 competition. However, the genetic or social emphasis on mating system can affect conclusions of studies (Coltman et al., 1999; Griffith, Owens, \& Thuman, 2002), especially 402 for sex-biased dispersal (Mabry et al., 2013). In particular, the social view of mating system 403 gives a greater importance to resources and it can also consider other factors of dispersal such 404 as tenure duration (Clutton-Brock \& Lukas, 2012; Graw, Lindholm, \& Manser, 2016) or 405 cooperation (Graw et al., 2016; Ridley, 2012). Despite our poor knowledge of the interplay of 
407

408

409

410

411

412

413

414

415

416

417

418

419

420

421

422

423

424

425

426

427

428

429

430

431

safely say that kin competition is universal as advocated by Lambin et al. (2001). Thus, our current study strengthens Dobson's (2013) message that there is a need for studies exploring the relationship between kin competition and sex-biased dispersal.

Acknowledgments

We thank two anonymous referees for helpful comments on the manuscript.

\section{References}

Alonso-Alvarez, C. (2006). Manipulation of primary sex-ratio: an updated review. Avian and Poultry Biology Reviews, 17, 1-20. doi:10.3184/147020606783437930

Bach, L. A., Thomsen, R., Pertoldi, C., \& Loeschcke, V. (2006). Kin competition and the evolution of dispersal in an individual-based model. Ecological Modelling, 192, 658666. doi:10.1016/j.ecolmodel.2005.07.026

Beirinckx, K., Van Gossum, H., Lajeunesse, M. J., \& Forbes, M. R. (2006). Sex biases in dispersal and philopatry: insights from a meta-analysis based on capture-mark-recapture studies of damselflies. Oikos, 113, 539-547. doi:10.1111/j.2006.0030-1299.14391.x

Bennett, V. J., Pack, S. M., Smith, W. P., \& Betts, M. G. (2013). Sex-biased dispersal in a rare butterfly and the implications for its conservation. Journal of Insect Conservation, 17(5), 949-958. doi:10.1007/s10841-013-9577-5

Benton, T. G., \& Bowler, D. E. (2012). Dispersal in invertebrates: influences on individual decisions. In J. Clobert, M. Baguette, T. G. Benton, \& J. M. Bullock (Eds.), Dispersal Ecology and Evolution (pp. 41-49). Oxford, U.K.: Oxford University Press.

Bonte, D., Van Dyck, H., Bullock, J. M., Coulon, A., Delgado, M., Gibbs, M., et al. (2012). Costs of dispersal. Biological Reviews, 8, 290-312. doi:10.1111/j.1469- 
433 Boudjemadi, K., Lecomte, J., \& Clobert, J. (1999). Influence of connetivity on demography

434

435 and dispersal in two contrasting habitats: an experimental approach. Journal of Animal Ecology, 68, 1207-1224. doi:10.1046/j.1365-2656.1999.00363.x

Chapple, D. G., \& Keogh, J. S. (2005). Complex mating system and dispersal patterns in a social lizard, Egernia whitii. Molecular Ecology, 14, 1215-1227. doi:10.1111/j.1365294X.2005.02486.x

Clobert, J., Danchin, E., Dhondt, A. A., \& Nichols, J. D. (2001). Dispersal. Oxford, U.K.: Oxford University Press.

Clobert, J., Le Galliard, J.-F., Cote, J., Meylan, S., \& Massot, M. (2009). Informed dispersal, heterogeneity in animal dispersal syndromes and the dynamics of spatially structured populations. Ecology Letters, 12, 197-209. doi:10.1111/j.1461-0248.2008.01267.x

Clobert, J., Massot, M., \& Le Galliard, J.-F. (2012). Multi-determinism in natal dispersal: the common lizard as a model system. In J. Clobert, M. Baguette, T. G. Benton, \& J. M. Bullock (Eds.), Dispersal Ecology and Evolution (pp. 29-40). Oxford, U.K.: Oxford University Press.

Clutton-Brock, T. H., \& Lukas, D. (2012). The evolution of social philopatry and dispersal in female mammals. Molecular Ecology, 21, 472-492. doi:10.1111/j.1365294X.2011.05232.x

Cockburn, A. (1989). Sex-ratio variation in marsupials. Australian Journal of Zoology, 37, 467. doi:10.1071/ZO9890467

Coltman, D. W., Bancroft, D. R., Robertson, A., Smith, J. A., Clutton-Brock, T. H., \& Pemberton, J. M. (1999). Male reproductive success in a promiscuous mammal: Behavioural estimates compared with genetic paternity. Molecular Ecology, 8, 11991209. doi:10.1046/j.1365-294X.1999.00683.x 
457 Comins, H. N., Hamilton, W. D., \& May, R. M. (1980). Evolutionarily stable dispersal 458 strategies. Journal of Theoretical Biology, 82(2), 205-230. doi:10.1016/0022$459 \quad 5193(80) 90099-5$

460 Dobson, F. S. (1982). Competition for mates and predominant juvenile male dispersal in mammals. Animal Behaviour, 30, 1183-1192. doi:10.1016/S0003-3472(82)80209-1

462 Dobson, F. S. (2013). The enduring question of sex-biased dispersal: Paul J. Greenwood's (1980) seminal contribution. Animal Behaviour, 85, 299-304. doi:10.1016/j.anbehav.2012.11.014

Frank, S. A. (1986). Dispersal polymorphisms in subdivided populations. Journal of Theoretical Biology, 122, 303-309. doi:10.1016/S0022-5193(86)80122-9

Gienapp, P., \& Merilä, J. (2011). Sex-specific fitness consequences of dispersal in Siberian 468 jays. Behavioral Ecology and Sociobiology, 65, 131-140. doi:10.1007/s00265-010-1017$\mathrm{X}$

Graw, B., Lindholm, A. K., \& Manser, M. B. (2016). Female-biased dispersal in the solitarily foraging slender mongoose, Galerella sanguinea, in the Kalahari. Animal Behaviour, Animal Behaviour, 28, 1140-1162. doi:10.1016/S0003-3472(80)80103-5

Griffith, S. C., Owens, I. P. F., \& Thuman, K. A. (2002). Extra pair paternity in birds: A review of interspecific variation and adaptive function. Molecular Ecology, 11, 21952212. doi:10.1046/j.1365-294X.2002.01613.x

Gros, A., Hovestadt, T., \& Poethke, H. J. (2008). Evolution of sex-biased dispersal: the role of sex-specific dispersal costs, demographic stochasticity, and inbreeding. Ecological Modelling, 219, 226-233. doi:10.1016/j.ecolmodel.2008.08.014 
reproductive success promotes the evolution of sex-biased dispersal. Theoretical Population Biology, 76, 13-18. doi:10.1016/j.tpb.2009.03.002

484

485

486

487

488

489

490

491

492

493

494

495

496

497

498

499

500

501

502

503

504

505

506

Gu, H., Hughes, J., \& Dorn, S. (2006). Trade-off between mobility and fitness in Cydia pomonella L. (Lepidoptera: Tortricidae). Ecological Entomology, 31, 68-74. doi:10.1111/j.0307-6946.2006.00761.x

Guillaume, F., \& Perrin, N. (2006). Joint evolution of dispersal and inbreeding load. Genetics, 173, 497-509. doi:10.1534/genetics.105.046847

Hamilton, W. D., \& May, R. M. (1977). Dispersal in stable habitats. Nature, 269, 578-581. doi:10.1038/269578a0

Johnson, M. L., \& Gaines, M. S. (1990). Evolution of dispersal: theoretical models and empirical tests using birds and mammals. Annual Review of Ecology and Systematics, 21, 449-480. doi:10.1146/annurev.es.21.110190.002313

Kokko, H., \& López-Sepulcre, A. (2006). From individual dispersal to species ranges: perspectives for a changing world. Science, 313, 789-791. doi:10.1126/science.1128566

Kubisch, A., Fronhofer, E. A., Poethke, H. J., \& Hovestadt, T. (2013). Kin competition as a major driving force for invasions. The American Naturalist, 181, 700-706. doi:10.1086/670008

Lagisz, M., Wolff, K., Sanderson, R. A., \& Laskowski, R. (2010). Genetic population structure of the ground beetle, Pterostichus oblongopunctatus, inhabiting a fragmented and polluted landscape: evidence for sex-biased dispersal. Journal of Insect Science (Online), 10, 105. doi:10.1673/031.010.10501

Lambin, X., Aars, J., \& Piertney, S. B. (2001). Dispersal, intraspecific competition, kin competition and kin facilitation: A review of the empirical evidence. In J. Clobert, E. Danchin, A. A. Dhondt, \& J. D. Nichols (Eds.), Dispersal (pp. 110-122). Oxford, U.K.: Oxford University Press. 
Lawson Handley, L. J., \& Perrin, N. (2007). Advances in our understanding of mammalian sex-biased dispersal. Molecular Ecology, 16, 1559-1578. doi:10.1111/j.1365294X.2006.03152.x

Lehmann, L., \& Balloux, F. (2007). Natural selection on fecundity variance in subdivided populations: Kin selection meets bet hedging. Genetics, 176, 361-377. doi:10.1534/genetics.106.066910

Leturque, H., \& Rousset, F. (2004). Intersexual competition as an explanation for sex-ratio and dispersal biases in polygynous species. Evolution, 58, 2398-2408. doi:doi: 10.1111/j.0014-3820.2004.tb00870.x

López-Uribe, M. M., Zamudio, K. R., Cardoso, C. F., \& Danforth, B. N. (2014). Climate, physiological tolerance and sex-biased dispersal shape genetic structure of Neotropical orchid bees. Molecular Ecology, 23, 1874-1890. doi:10.1111/mec.12689

Mabry, K. E., Shelley, E. L., Davis, K. E., Blumstein, D. T., \& van Vuren, D. H. (2013). Social mating system and sex-biased dispersal in mammals and birds: A phylogenetic analysis. PLoS ONE, 8(3), e57980. doi:10.1371/journal.pone.0057980

Markow, T. A., \& Castrezana, S. (2000). Dispersal in cactophilic Drosophila. Oikos, 89, 378386. doi:10.1034/j.1600-0706.2000.890219.x

Massot, M., Clobert, J., Lecomte, J., \& Barbault, R. (1994). Incumbent advantage in common lizards and their colonizing ability. Journal of Animal Ecology, 63, 440. doi: $10.2307 / 5560$

Massot, M., Clobert, J., Pilorge, T., Lecomte, J., \& Barbault, R. (1992). Density dependence in the common lizard: Demographic consequences of a density manipulation. Ecology, $73,1742-1756$.

Matthysen, E. (2012). Multicausality of dispersal: a review. In J. Clobert, M. Baguette, T. G. Benton, \& J. M. Bullock (Eds.), Dispersal Ecology and Evolution (pp. 3-18). Oxford, 
Murrell, D. J., Travis, J. M. J., \& Dytham, C. (2002). The evolution of dispersal distance in spatially-structured populations. Oikos, 97, 229-236. doi:10.1034/j.16000706.2002.970209.x

Nagy, M., Günther, L., Knörnschild, M., \& Mayer, F. (2013). Female-biased dispersal in a bat with a female-defence mating strategy. Molecular Ecology, 22, 1733-1745. doi:10.1111/mec.12202

Nespolo, R. F., Roff, D. A., \& Fairbairn, D. J. (2008). Energetic trade-off between maintenance costs and flight capacity in the sand cricket (Gryllus firmus). Functional Ecology, 22, 624-631. doi:10.1111/j.1365-2435.2008.01394.x

Nevoux, M., Arlt, D., Nicoll, M., Jones, C., \& Norris, K. (2013). The short- and long-term fitness consequences of natal dispersal in a wild bird population. Ecology Letters, 16, 438-445. doi:10.1111/ele.12060

Ode, P. J., Antolin, M. F., \& Strand, M. R. (1998). Differential dispersal and female-biased sex allocation in a parasitic wasp. Ecological Entomology, 23, 314-318. doi:10.1046/j.1365-2311.1998.00134.x

Parvinen, K., Dieckmann, U., Gyllenberg, M., \& Metz, J. A. J. (2003). Evolution of dispersal in metapopulations with local density dependence and demographic stochasticity. Journal of Evolutionary Biology, 16, 143-153. doi:10.1046/j.1420-9101.2003.00478.x

Perrin, N., \& Goudet, J. (2001). Inbreeding, kinship, and the evolution of natal dispersal. In J. Clobert, E. Danchin, A. A. Dhondt, \& J. D. Nichols (Eds.), Dispersal (pp. 123-142). Oxford, U.K.: Oxford University Press.

Perrin, N., \& Lehmann, L. (2001). Is sociality driven by the costs of dispersal or the benefits of philopatry? A role for kin-discrimination mechanisms. The American Naturalist, 158, 471-483. doi:10.1086/323114 
Perrin, N., \& Mazalov, V. (1999). Dispersal and inbreeding avoidance. The American Naturalist, 154(3), 282-292. doi:10.1086/303236

Perrin, N., \& Mazalov, V. (2000). Local competition, inbreeding, and the evolution of sexbiased dispersal. The American Naturalist, 155, 116-127. doi:10.1086/303296

Poethke, H. J., Pfenning, B., \& Hovestadt, T. (2007). The relative contribution of individual and kin selection to the evolution of density-dependent dispersal rates. Evolutionary Ecology Research, 9, 41-50.

Reynolds, J. D. (1996). Animal breeding systems. Trends in Ecology \& Evolution, 11, 68-72.

Ridley, A. R. (2012). Invading together: The benefits of coalition dispersal in a cooperative bird. Behavioral Ecology and Sociobiology, 66, 77-83. doi:10.1007/s00265-011-1255-6

Ronce, O. (2007). How does it feel to be like a rolling stone? Ten questions about dispersal evolution. Annual Review of Ecology, Evolution, and Systematics, 38, 231-253. doi:10.1146/annurev.ecolsys.38.091206.095611

Roze, D., \& Rousset, F. (2005). Inbreeding depression and the evolution of dispersal rates: a multilocus model. The American Naturalist, 166, 708-721. doi:10.1086/497543

Soulsbury, C. D., Baker, P. J., Iossa, G., \& Harris, S. (2008). Fitness costs of dispersal in red foxes (Vulpes vulpes). Behavioral Ecology and Sociobiology, 62, 1289-1298. doi:DOI $10.1007 / \mathrm{s} 00265-008-0557-9$

Taylor, P. D. (1988). An inclusive fitness model for dispersal of offspring. Journal of Theoretical Biology, 130, 363-378. doi:10.1016/S0022-5193(88)80035-3

Travis, J. M. J., \& Dytham, C. (1998). The evolution of dispersal in a metapopulation: a spatially explicit, individual-based model. Proceedings of the Royal Society B: Biological Sciences, 265, 17-23. doi:10.1098/rspb.1998.0258

Walther, G.-R., Post, E., Convey, P., Menzel, A., Parmesan, C., Beebee, T. J. C., ... Bairlein, F. (2002). Ecological responses to recent climate change. Nature, 416, 389-395. 
583 West, S. A., Shuker, D. M., \& Sheldon, B. C. (2005). Sex-ratio adjustment when relatives interact: a test of constraints on adaptation. Evolution, 59, 1211-1228. doi:10.1554/04158

586 White, J. W., Rassweiler, A., Samhouri, J. F., Stier, A. C., \& White, C. (2014). Ecologists

587 should not use statistical significance tests to interpret simulation model results. Oikos,

588 123, 385-388. doi:10.1111/j.1600-0706.2013.01073.x

589 Wild, G., \& Taylor, P. D. (2004). Kin selection models for the co-evolution of the sex-ratio 590 and sex-specific dispersal. Evolutionary Ecology Research, 6, 481-502.

591 
594 Fig. 1. Dispersal rate at equilibrium for females (circles) and males (squares) in the 595 unshuffled model (filled symbols) and shuffled (i.e. without genetic structure) model (open 596 symbols) for the three mating systems studied. (a) Monogamy, (b) monandry and (c) 597 polyandry. Parameters were fixed to their base value as in Table 1. Error bars indicate 95\% 598 confidence interval built by bootstrapping the results of 1000 replicate simulation runs. 599

Fig. 2. Dispersal rate at equilibrium for females (circles) and males (squares) as a function of 601 heterogeneity in patch quality $(\sigma)$ for the three mating systems studied. (a) Monogamy, (b) 602 monandry and (c) polyandry. Filled symbols: unshuffled model; open symbols: shuffled (i.e. 603 without genetic structure) model. Other parameters were fixed to their base value as in Table 604 1. The $95 \%$ confidence intervals built by bootstrapping the results of 1000 replicate 605 simulation runs are smaller than the symbols' height (which was set to 0.05 dispersal rate units to ensure visibility). Values shown in Fig. 1 are highlighted by vertical dotted lines.

607

608 Fig. 3. Dispersal rate at equilibrium for males (grey) and females (black) plotted against 609 homozygosity cost on fecundity for the three mating systems studied. (a) Monogamy, (b) monandry and (c) polyandry. Heterogeneity in patch quality $(\sigma)$ was fixed to 1 , and other

611 parameters were fixed to their base value as in Table 1. Line width indicates 95\% confidence 612 interval built by bootstrapping the results of 100 replicate simulation runs.

614 Fig. 4. Dispersal rate at equilibrium for males (grey) and females (black) for a range of male dispersal costs and for the three mating systems studied. (a) Monogamy, (b) monandry and (c) 616 polyandry. Female dispersal cost $(\mu)$ was fixed to 0.1 , and other parameters were fixed to their 
617 base value as in Table 1. The vertical dotted line shows equality of dispersal costs between

618 males and females. Line width indicates 95\% confidence interval built by bootstrapping the 619 results of 1000 replicate simulation runs.

620

621 Fig. 5. Dispersal rate at equilibrium for males (grey) and females (black) when the sex ratio 622 (proportion of males) varies for the three mating systems studied. (a) Monogamy, (b) 623 monandry and (c) polyandry. Other parameters were fixed to their base value as in Table 1. 624 Line width indicates $95 \%$ confidence interval built by bootstrapping the results of 1000 625 replicate simulation runs.

626

627 Fig. A1. Mean relatedness index before dispersal between individuals of the same patches 628 (upward triangle) and from different patches (downward triangle) in the unshuffled model 629 (filled symbols) and the shuffled (i.e. without genetic structure) model (open symbols) for the 630 three mating systems studied. (a) Monogamy, (b) monandry and (c) polyandry. Parameters 631 were fixed to their base value as in Table 1. Error bars indicate 95\% confidence interval built 632 by bootstrapping the results of 1000 replicate simulation runs.

633

634 Fig. A2. Between-patch coefficient of variation in mean per capita reproductive success for 635 females (circles) and males (squares) in the unshuffled model (filled symbols) and the 636 shuffled (i.e. without genetic structure) model (open symbols) for the three mating systems 637 studied. (a) Monogamy, (b) monandry and (c) polyandry. Parameters were fixed to their base 638 value as in Table 1. Error bars indicate 95\% confidence interval built by bootstrapping the 639 results of 1000 replicate simulation runs. 
641 Fig. A3. Dispersal rate at equilibrium for males (grey) and females (black) in (a, d) monandry 642 and (e, h) polyandry with increasing heterogeneity in patch quality from (a) to (d) and from 643 (e) to (h): (a),(e): $\sigma=0$; (b),(f): $\sigma=0.5$; (c),(g): $\sigma=1$; (d),(h): $\sigma=2$. Other parameters 644 were fixed to their base value as in Table 1. Line width indicates $95 \%$ confidence interval 645 built by bootstraping the results of 100 replicate simulation runs.

646

647 Fig. A4. Density-independent dispersal rate at equilibrium for females (circles) and males 648 (squares) in the standard (black) and the shuffled (blank) models for the three mating systems 649 studied. (a) Monogamy, (b) monandry and (c) polyandry. Parameters were fixed to their base 650 value as in Table 1 . The 95\% confidence intervals built by bootstrapping the results of 1000 651 replicate simulation runs are smaller than the symbols' height (which was set to 0.05 dispersal 652 rate units to ensure visibility). We tested density-independent dispersal simply by setting $653 d p_{i}=d s_{i}$ : the probability of dispersal of an individual is equal to the value of its adaptive 654 trait, the dispersal strategy. 
656 Table 1: Simulation parameters

\begin{tabular}{lll}
\hline Name & Symbol & Base value \\
\hline Patch capacity & $K$ & 100 \\
Number of patches & $n_{\text {patch }}$ & 100 \\
Dispersal mortality & $\mu$ & 0.1 \\
Mean patch quality & $\lambda$ & 2 \\
Heterogeneity in patch quality & $\sigma$ & 0.5 \\
Intensity of density dependence & $\beta$ & 1 \\
Primary sex ratio & $s r_{\mathrm{b}}$ & 0.5 \\
Homozygosis penalty coefficient & $\rho$ & 0 \\
Mutation frequency on dispersal alleles & $f_{\mathrm{s}}$ & 0.001 \\
Mutation standard deviation on dispersal alleles & $s d_{\mathrm{s}}$ & 0.05 \\
Mutation frequency on neutral alleles & $f_{\mathrm{n}}$ & 0.001 \\
\hline
\end{tabular}

657

658 